Research Article

\title{
The Effect of Parallel-Axis Helix Gear Pair on Wind Turbine Gearbox Vibration Control
}

\author{
Zheng Li $\mathbb{D},{ }^{1}$ Tianhe Zhang, ${ }^{1}$ Yang Chen, ${ }^{2}$ and Lijuan Song ${ }^{1}$ \\ ${ }^{1}$ School of Naval Architecture and Marine Engineering, Dalian Maritime University, Dalian, China \\ ${ }^{2}$ Dalian Scientific Test and Control Technology Institute, Dalian, China \\ Correspondence should be addressed to Zheng Li; zhengli@dlmu.edu.cn
}

Received 15 March 2020; Revised 20 June 2020; Accepted 26 July 2020; Published 14 August 2020

Academic Editor: Cristina Castejón

Copyright (c) 2020 Zheng Li et al. This is an open access article distributed under the Creative Commons Attribution License, which permits unrestricted use, distribution, and reproduction in any medium, provided the original work is properly cited.

This article studies the effects of some basic parameters of a parallel-axis helix gear stage on wind turbine gearbox vibration in a case study: a multibody dynamic model is constructed to simulate the drive train of a faulted multistage wind turbine gearbox with serious vibrations. The significant vibration behaviour of the drive train for typical excitations is calculated, and the results according to specified geometric parameters of the gears are analysed in detail to investigate effective solutions for vibration reduction. The results indicate that the helix angle and numbers of teeth of a gear pair are the most significant factors for solving the problem. The effectiveness of the proposed solutions and relevant mechanisms are discussed and validated by a prototype vibration test.

\section{Introduction}

The dynamic performance of a gearbox is a kind of characteristic that can potentially affect reliability in an obvious way, so the vibration of the gearbox system should be evaluated critically. An obvious or potential fault will increase the failure risk of gearbox and cause unexpected resource waste due to mandatory maintenance. A common theory which is widely accepted for the gearbox design is that the most significant performance of gearbox should be the fatigue life and static strength of the rotating components such as meshing gears, shafts, and bearings, so the mechanical properties of the transmission parts must satisfy the particular design requirements. Actually, some problems of a faulted gearbox always derive from uncontrollable dynamic responses to particular excitations on typical work conditions, and this principle is frequently mentioned and reported in the relevant research literature [1-3]. To research the mechanism of gearbox vibration faults and find effective solutions, many simulation models have been proposed to study the influence of typical boundary conditions on gearbox vibrations. The common modelling objective is to simulate the gear meshing properties, because the excitation effects on the gearbox always derive from an internal cause (gear meshing) and an external cause (dynamic load) [4]. For studies on a wind turbine gearbox, multibody simulation models are frequently applied, because the gearbox is a coupled multibody system with complicated boundary conditions, and the simulation of the planetary gear stage should be the most significant in the modelling. Parra and Vicuna [5] proposed an analytical model and a lumpedparameter model that were constructed on the basis of a single planetary gear stage to investigate planetary gearbox vibration on different fault conditions. He et al. [6] developed a set of rigid-flexible coupling dynamic models of a single-stage planetary gear train with a floating sun gear shaft. Single planetary gear stage modelling can simulate the gear meshing stiffness of a planetary gear train and should be considered the foundation of the model, which is constructed on the basis of a coupled multistage drive train of a wind turbine gearbox. According to single planetary gear stage modelling, Zhao and Ji [7] developed a multibody dynamic model of a wind turbine gearbox with two planetary gear stages and one parallel-axis gear stage to research nonlinear torsional vibrations during wind turbine gearbox operation. Al-Hamadani et al. [8] constructed a coupled 
model of a wind turbine gearbox by using MATLAB/ Simulink to analyse the gear load variation and the system vibration on two typical transient loading conditions (normal operating and shutdown). Additionally, the gearbox model can also be considered as a subsystem of an integrated wind turbine system and combined with other functional components of the wind turbine such as the blades and tower; models of the integrated wind turbine system are always constructed to study some special excitations that do not influence the gearbox directly, such as the platform motion of an offshore wind turbine $[9,10]$. The conclusions derived from the model of a coupled multistage gear train can describe the dynamic performance of a wind turbine gearbox practically due to the consideration of the coupling parameters between coupled gear stages. The model and research strategy are significant for solving vibration faults of a wind turbine gearbox.

Some studies on fault diagnosis for gearboxes have validated that the failure of any component in a drive train causes a particular variation in the performance. The source of a gearbox vibration can be reflected by signals of vibration behaviour $[5,6,11]$ and can be captured and displayed by a particular expert system or procedure [12], so it is feasible to research the dynamic performance of a wind turbine drive train by analysing the different vibration signals, such as the time-domain spectrum and meshing frequency spectrum $[13,14]$. The results derived from various analytical methods and the relevant discussions indicated that many factors can influence the vibration of a wind turbine gearbox. Wei et al. [15] showed that the mesh stiffness, transmission error, and mesh damping are significant for the vibration behaviour of a wind turbine transmission system. Wang et al. [16] pointed out that a gear micromodification scheme could significantly influence the vibration behaviour. Yi, Huang, and Shi [17] validated that the modal properties of a wind turbine drive train are relevant to the vibration behaviour of the gearbox. Additionally, helix gears are widely applied in wind turbine gearbox design, and the influence of particular geometric parameters, such as the helix angle, on the vibration behaviour of the gearbox is also frequently investigated. Tatar et al. [18] indicated that the vibrations of gear shafts were coupled in the helical gearing configuration due to the gear helix angle. Lei et al. [19] solved the noise and vibration problem of an electric bus gearbox and presented an optimal microgeometry modification algorithm to reduce noise excitation sources such as the transmission error and maximum tooth surface load. Hiremath and Venkataram [20] proposed that design modifications of the gearbox should be carried out to avoid resonance. The conclusions of the abovementioned literature supplies a theoretical foundation for the research in this article. It is obvious that the geometric parameters of the gears are significant for the fault diagnosis and vibration behaviour optimization of a wind turbine gearbox.

Many studies on wind turbine gearbox vibration have proposed various reliable models, relative factors, and critical analytical methods for gearbox fault diagnosis, but the integrated influences of multiple factors on wind turbine gearbox vibration are reported insufficiently owing to the various limitations of the simulation models; additionally, conclusions regarding specific optimal design methodologies for vibration reduction on the basis of modal optimization by altering the geometric parameters of the gears are also scarce. According to the objectives of this research, the simulation model is constructed according to the structure of a sampled multistage wind turbine gearbox. The boundary conditions that are validated to be relevant to gearbox vibration are considered comprehensively, which include the flexibilities of the shafts, gears, and housings; the stiffness and damping of the bearings, couplings, and gear meshing; the mass and inertia of all components in the drive train; and the backlash of the gear pairs. This article analyses the relationship between gearbox vibrations and the geometric parameters of a helix gear pair with consideration of the eigenfrequencies and resonance performance of the drive train and proposes a practical optimal solution for vibration reduction. The effectiveness is validated by experimental discussions.

\section{Modelling}

2.1. Question Description. The research object in this article is a wind turbine gearbox with traditional transmission concept and structure which has two spur planetary stages and one parallel-axis helix stage. Some basic technical parameters of the wind turbine are listed in Table 1.

The faulted gearbox exhibited terrible translational vibrations on the output shaft with the highest rotation velocity (HSS) when the wind turbine was running on rated work condition. According to the validation by vibration test, the experimental results which are listed in Table 2 indicated that the acceleration of HSS seriously exceeded mandatory vibration limitation $\left(7.5 \mathrm{~m} / \mathrm{s}^{2}\right.$ in VDI 3834$)$. Figure 1 illustrates the position of the acceleration sensor and modelling logic of the simulation.

To investigate the main source of faulted gearbox vibrations and research effective solution, a coupled multibody dynamic model of the sample gearbox is constructed for the simulating analysis. This model is proposed to analyse the dynamic behaviour of the faulted gearbox and determine the factors which are significantly relevant to HSS vibration generation. A practical optimization scheme is presented as a reliable solution according to the simulation results, and the detailed discussions and relevant validations are presented.

2.2. Multibody Dynamic Model. The dynamic model is constructed by using SIMPACK, which is professional software for carrying out coupled multibody dynamic simulation. According to the modelling logic illustrated in Figure 1, the modelling assignments should consider following principles:

(a) The model shall contain all of rotating parts and stationary housings; some basic characteristic of the components such as mass and inertia must be defined precisely; all of the shafts shall be constructed as elastic components to consider the integrated stiffness of the drive train; the rear housing is constructed as extraflexible bodies because it is directly relevant to the vibration behaviour of the parallel-axis helix gear stage (HSS). 
TABLE 1: Basic technical parameters of wind turbine.

\begin{tabular}{lc}
\hline Rated Power & $1.71 \mathrm{MW}$ \\
Rated input torque & $1021 \mathrm{kNm}$ \\
Rated input rotation velocity & $16 \mathrm{rpm}$ \\
Ratio of the gearbox & 112.6 \\
Output rotation velocity range & $1015-2060 \mathrm{rpm}$ \\
\hline
\end{tabular}

TABLE 2: Original vibration test results.

\begin{tabular}{lc}
\hline Direction & Acceleration $\left(\mathrm{m} / \mathrm{s}^{2}\right)$ \\
\hline Axial & 8.18 \\
Vertical & 21.65 \\
Radial & 15.29 \\
\hline
\end{tabular}

(b) The model shall adequately consider the connective stiffness between two coupled components, such as couplings, interference fit, spline, and elastic support. The bearing stiffness must be considered at least in the form of a matrix, but the nonlinear stiffness spectrum of the HSS bearings should be considered to improve the accuracy of the vibration behaviour results.

(c) The geometric parameters of all gears shall be defined precisely according to the design scheme of the drive train, and the meshing stiffness and damping of all gear pairs (including one parallel-axis helix stage and two spur planetary stages) need to be considered as precise as possible.

(d) The rated load and rotation velocity shall be considered as the input/output parameters of the simulation because the vibration fault is significantly obvious on rated work condition.

In a coupled multibody drive train, the motion differential equation can be derived by analysing the actual forces acting on the components. The composition of the force acting on a single mass $m_{a}$ consists of an elastic force, an inertial force, and a damping force. Under the assumption that the displacement function of a mass $m_{a}$ is $q(t)$, the three kinds of force should be as follows:

$$
\left\{\begin{array}{l}
F_{K}=K_{a} \cdot q(t), \\
F_{I}=m_{a} \cdot \ddot{q}(t), \\
F_{c}=C_{a} \cdot \dot{q}(t) .
\end{array}\right.
$$

If there are $n$ components in a multibody drive train and the displacement function of each mass is $q_{b}(t)(b=1,2, \ldots$, $n)$, the three kinds of force acting on mass $m_{a}$ should be superposed on the basis of the superposition principle:

$$
\left\{\begin{array}{l}
F_{K}=\sum_{b=1}^{n} K_{a b} \cdot q_{b}(t), \\
F_{I}=\sum_{b=1}^{n} m_{a b} \cdot \ddot{q}_{b}(t), \\
F_{c}=\sum_{b=1}^{n} C_{a b} \cdot \dot{q}_{b}(t) .
\end{array}\right.
$$

According to the D'Alembert principle, the elastic force, inertial force, damping force, and exciting force acting on mass $m_{a}$ constitute an equilibrium force system. The equation is

$$
\sum_{\mathrm{b}=1}^{n} m_{a b} \ddot{q}_{b}(t)+\sum_{b=1}^{n} C_{a b} \dot{q}_{b}(t)+\sum_{b=1}^{n} K_{a b} q_{b}(t)=Q_{a}(t) .
$$

Equation (3) is established for each mass in the multibody drive train, and the subscript $a$ should be assigned from 1 to $n$, which means there are $n$ equations based on equation (3) for this drive train. Consequently, the equations can be written as follows:

$$
\begin{array}{r}
\sum_{b=1}^{n}\left(m_{a b} \ddot{q}_{b}(t)+C_{a b} \dot{q}_{b}(t)+K_{a b} q_{b}(t)\right)=Q_{a}(t), \\
(a=1,2, \cdots, n) .
\end{array}
$$

Equation (4) is the motion differential equation of a coupled multibody drive train with $n$ components; it is a second-order linear differential equation with constant coefficients and can be integrated into matrix form:

$$
\begin{aligned}
{[m]\{\ddot{q}(t)\}+[C]\{\dot{q}(t)\}+[K]\{q(t)\} } & =\{Q(t)\}, \\
\{q(t)\} & =\left\{q_{1}(t), q_{2}(t), \cdots q_{n}(t)\right\}^{T}, \\
\{Q(t)\} & =\left\{Q_{1}(t), Q_{2}(t), \cdots Q_{n}(t)\right\}^{T} .
\end{aligned}
$$

In equation (5), $[m],[C]$, and $[K]$ are the matrices of the mass, damping, and stiffness, and the boundary conditions define the dynamic performance of the drive train.

The wind turbine gearbox drive train is a representative multibody system, and all coefficients of the motion differential equation can also be generalized in a matrix. The most difficult question for the simulation is the analytical modelling of the planetary gear system, which is an important theoretical basis for constructing the multibody model of a wind turbine gearbox drive train.

Figure 2 indicates the analytical modelling of the planetary gear system in the faulted wind turbine gearbox [21]. Figure 3 illustrates drive principle of the faulted gearbox [22], so the integrated meshing forces of each planetary stage can be calculated by the following equations:

$$
\left\{\begin{array}{l}
X_{1 s p}=\cos \alpha_{1 s} \sum_{j=1}^{3} F_{1 s p j} \\
X_{1 \mathrm{r} p}=\cos \alpha_{1 p} \sum_{j=1}^{3} F_{1 r p j} \\
X_{2 s p}=\cos \alpha_{2 s} \sum_{j=1}^{3} F_{2 s p j} \\
X_{2 \mathrm{r} p}=\cos \alpha_{2 p} \sum_{j=1}^{3} F_{2 r p j}
\end{array}\right.
$$

Figure 3 also illustrates the components in each stage. According to the D'Alembert principle, equation (3) of each 
component in the wind turbine gearbox drive train can be established on the basis of the gearbox structure and relevant parameters such as the mass (or rotary inertia), stiffness, and damping of the geometries and couplings: follows:

The equations of motion for stage 1-planetary are as

$$
\left\{\begin{array}{l}
m_{1 c} \ddot{u}_{1 c}+C_{1 c} \frac{\dot{u}_{1 c}}{r_{1 c}}+K_{1 c} \frac{u_{1 c}}{r_{1 c}}=\frac{T_{i n}}{r_{1 c}}+X_{1 s p}-X_{1 r p} \\
m_{1 s} \ddot{u}_{1 s}+C_{1 s 2 c} \frac{\dot{u}_{1 s}}{r_{1 s}}+K_{1 s 2 c} \frac{u_{1 s}}{r_{1 s}}=-\sum_{j=1}^{3} F_{1 s p j} \\
m_{1 p 1} \ddot{u}_{1 p 1}=-\left(F_{1 s p 1}+F_{1 r p 1}\right) \\
m_{1 p 2} \ddot{u}_{1 p 2}=-\left(F_{1 s p 2}+F_{1 r p 2}\right) \\
m_{1 p 3} \ddot{u}_{1 p 3}=-\left(F_{1 s p 3}+F_{1 r p 3}\right) .
\end{array}\right.
$$
follows:

The equations of motion for stage 2-planetary are as

$$
\left\{\begin{array}{l}
m_{2 c} \ddot{u}_{2 c}+C_{2 c} \frac{\dot{u}_{2 c}}{r_{2 c}}+K_{2 c} \frac{u_{2 c}}{r_{2 c}}=\frac{T_{i n}}{i_{1} r_{2 c}}+X_{2 s p}-X_{2 r p}, \\
m_{2 s} \ddot{u}_{2 s}+C_{2 s 3 w} \frac{\dot{u}_{2 s}}{r_{2 s}}+K_{2 s 3 w} \frac{u_{2 s}}{r_{2 s}}=-\sum_{j=1}^{3} F_{2 s p j}, \\
m_{2 p 1} \ddot{u}_{2 p 1}=-\left(F_{2 s p 1}+F_{2 r p 1}\right), \\
m_{2 p 2} \ddot{u}_{2 p 2}=-\left(F_{2 s p 2}+F_{2 r p 2}\right), \\
m_{2 p 3} \ddot{u}_{2 p 3}=-\left(F_{2 s p 3}+F_{2 r p 3}\right) .
\end{array}\right.
$$
follows:

The equations of motion for stage 3-parallel are as

$$
\left\{\begin{array}{l}
m_{3 w} \ddot{u}_{3 w}+C_{2 s 3 w} \frac{\Delta \dot{\theta}_{2 s 3 w}}{r_{3 w}}+K_{2 s 3 w} \frac{\Delta \theta_{2 s 3 w}}{r_{3 w}}=\frac{T_{i n}}{f_{1} f_{2} r_{3 w}}-F_{3 w h s} \\
\Delta \dot{\theta}_{2 s 3 w}=\frac{\dot{u}_{3 w}}{r_{3 w}}-\frac{\dot{u}_{2 s}}{r_{2 s}}, \\
\Delta \theta_{2 s 3 w}=\frac{u_{3 w}}{r_{3 w}}-\frac{u_{2 s}}{r_{2 s}}, \\
m_{3 h s} \ddot{u}_{3 h s}=-\left(\frac{T_{\text {out }}}{r_{3 h s}}+F_{3 w h s}\right) .
\end{array}\right.
$$

Theoretically, according to equation (5), the motion differential equation of a multibody drive train could be integrated into matrix form with the concerned boundary conditions as follows:

$$
[m][\ddot{u}]+[C][\dot{u}]+[K][u]=[F] .
$$

Equation (10) indicates that the explicit responses of component acceleration under established excitation can be calculated by solving the motion differential equation set of the gearbox drive train. The results are discussed in the following sections, and the analytical solutions of the equation set are considered as the predictions of drive train vibration.

\section{Multibody Dynamic Simulations}

The multibody dynamic simulation is carried out for modal analysis and vibration behaviour calculation. According to the multibody dynamic principle, the eigenfrequencies and the translational vibration behaviour of the gearbox drive train are relevant to bearing stiffness. Many conventional approaches prefer to assume that the stiffness of the bearings applied in the gearbox are constant and then carry out modal analysis on a stationary drive train. Actually, the bearing stiffness should be significantly affected by working load, which means that the eigenfrequencies and the vibration behaviour should be analysed and calculated on particular work conditions. In this research, the actual stiffness spectrum of the HSS bearings derived from bearing manufacturing is considered, and the modal analysis is carried out considering the dynamic equilibrium of the gearbox drive train on rated work conditions.

3.1. Original Model. The original model is the model of the gearbox drive train constructed on the basis of the original design parameters. The reliability of the original model should be validated by an experimental approach. The frequency spectra of the translational accelerations of the sensor position are illustrated in the following figures to indicate the most significant excitation frequency.

Figures 4-6 indicate that the significant excitation frequency of the HSS translational acceleration responses is $937.9 \mathrm{~Hz}$, and all of the peak translational accelerations, especially the vertical acceleration, obviously exceed the vibration limitation. The distribution of the acceleration responses consists of the actual situation that is absolutely described by the fault report. According to Newton's second law of motion-force and acceleration, the serious acceleration response will cause an additional vibration load when the gearbox is running on work conditions. The vibration load must be limited significantly; otherwise, it would cause serious fatigue faults for the gearbox drive train. To compare the simulation results with the experimental results, the data indicate that the theoretical and experimental HSS accelerations are basically in agreement (see Table 3 ). The errors between the theoretical and experimental accelerations are considered to be caused by some boundary condition 

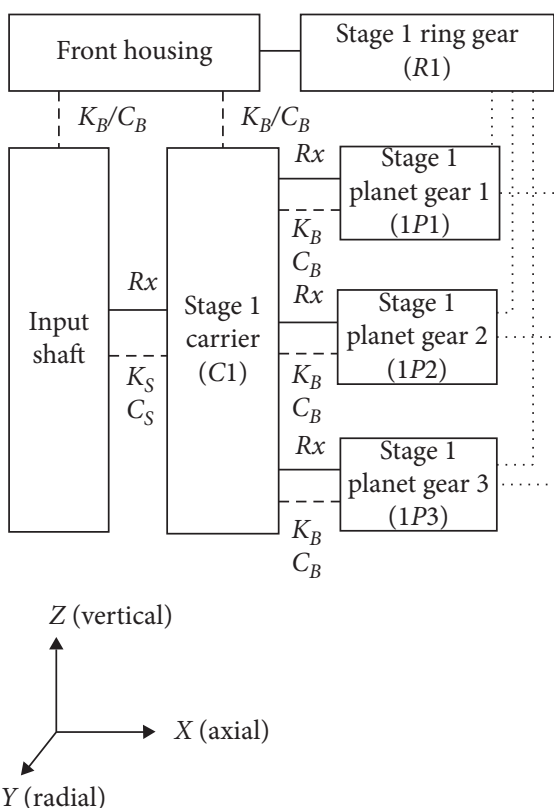
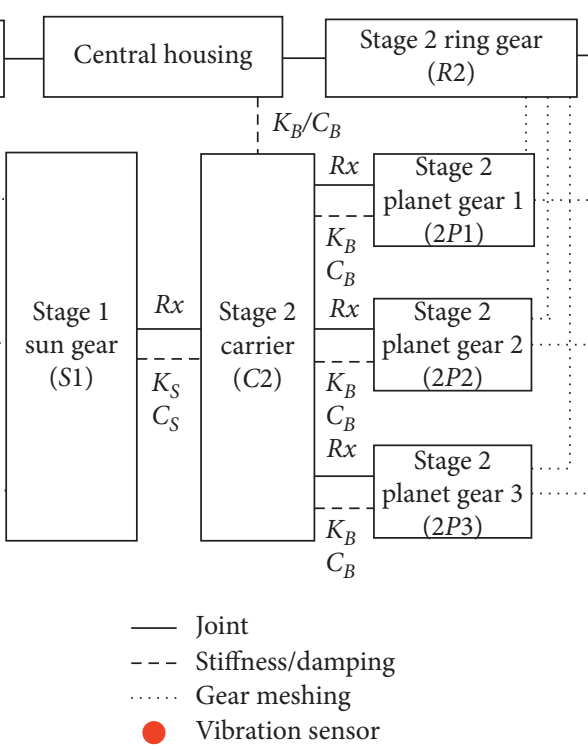

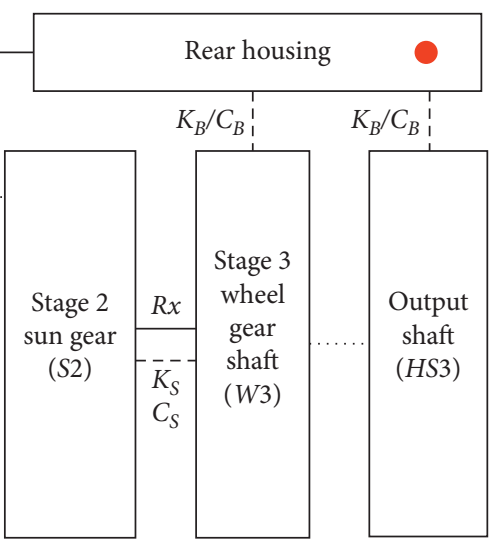

$T x, T y, T z:$ translation

$R x, R y, R z$ : rotation

$K_{B} / C_{B}$ : bearig stiffness/damping

$K_{S} / C_{S}:$ spline stiffness/damping

Figure 1: Position of the acceleration sensor and modelling logic of the simulation.

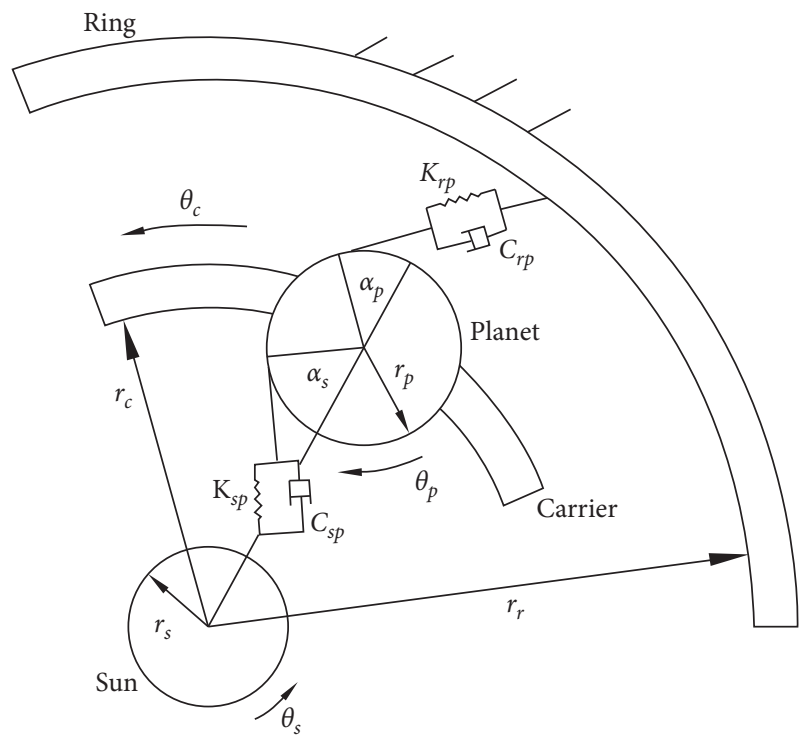

Figure 2: Analytical modelling of the planetary gear system [21].

deviations between theoretical simulation model and practical test bench, and the reliability of the simulation model can be validated according to the comparisons. To investigate the source of the serious vibration of the faulted wind turbine gearbox, the simulation results of the original model are considered as the fundamental data. The further discussions are presented on the basis of the acceleration responses and gear parameters of the original model.

Generally, the excitation frequency should not be allowed to approach any eigenfrequency to avoid the risk of resonance. The eigenfrequencies of the gearbox drive train can be obtained by a modal analysis, and the particular meshing frequency of the gear pair can derive from rotating

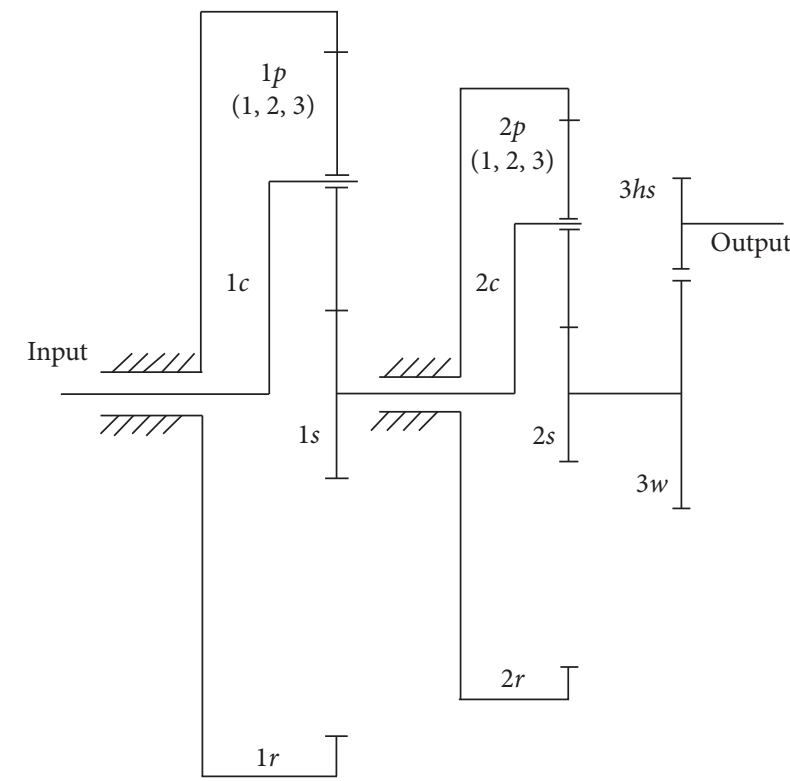

FIgURE 3: Drive principle of the faulted gearbox [22].

velocities and the numbers of teeth. According to the research purpose of this article, it is necessary to concern the meshing frequency of the parallel-axis helix stage on rated work conditions. The equation for calculating the gear meshing frequency is as follows:

$$
f_{\text {mesh }}=\frac{n_{a} Z_{a}}{60}=\frac{n_{b} Z_{b}}{60} .
$$

According to the basic design parameters of the faulted wind turbine gearbox, the original module of the helix stage is $7 \mathrm{~mm}$, and the number of teeth $Z_{a} / Z_{b}$ is $127 / 31$. The mesh frequencies on different work conditions are listed in Table 4. 


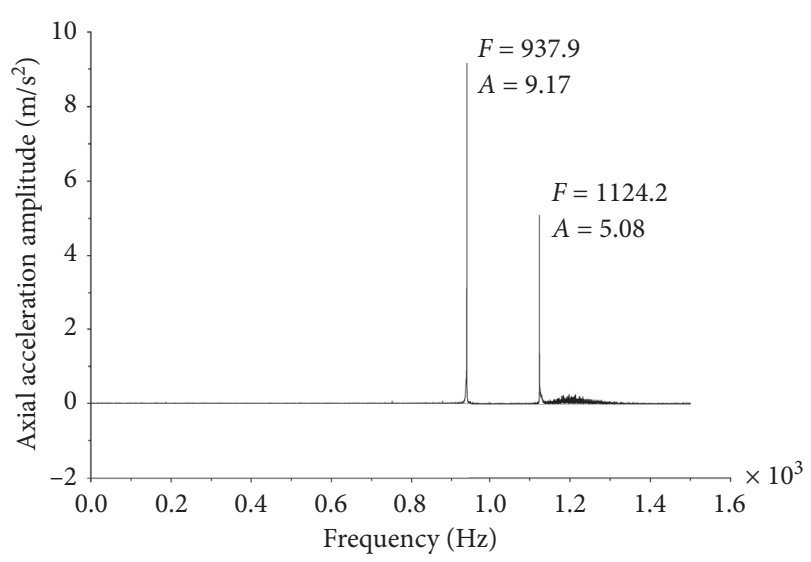

Figure 4: Axial acceleration of the original model.

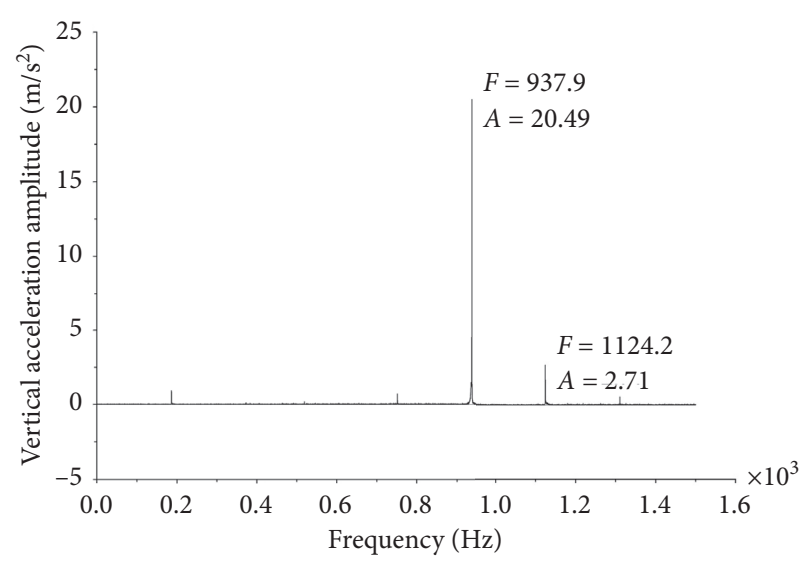

FIgURE 5: Vertical acceleration of the original model.

Table 4 indicates that the theoretical meshing frequency of the parallel-axis helix stage for the rated rotation velocity is $933.47 \mathrm{~Hz}$, which is very close to $937.9 \mathrm{~Hz}$, and the deviation rate is less than $1 \%$. This means that the significant excitation frequency in Figures 4-6 is the meshing frequency of the parallel-axis helix stage, and the faulted HSS vibration must derive from the helix gear meshing. To compare with the excitation frequencies, a modal analysis based on the original model should be carried out to obtain eigenfrequencies of the drive train (the drive train must achieve dynamic equilibrium on rated work conditions). According to the motion differential equations of the multibody drive train, the undamped free vibration equation can be concluded as

$$
\left(\omega_{i}^{2}[m]-[K]\right) \varphi_{i}=0 .
$$

To consider the damping coefficient $\xi_{i}$, the damped eigenfrequencies can be calculated according to undamped eigenfrequencies by the following equation:

$$
\omega_{d i}=\omega_{i} \sqrt{1-\xi_{i}^{2}} \text {. }
$$

Theoretically, a multibody system has numerous eigenfrequencies that can be obtained by a modal calculation. For the case study in this research, it is necessary to

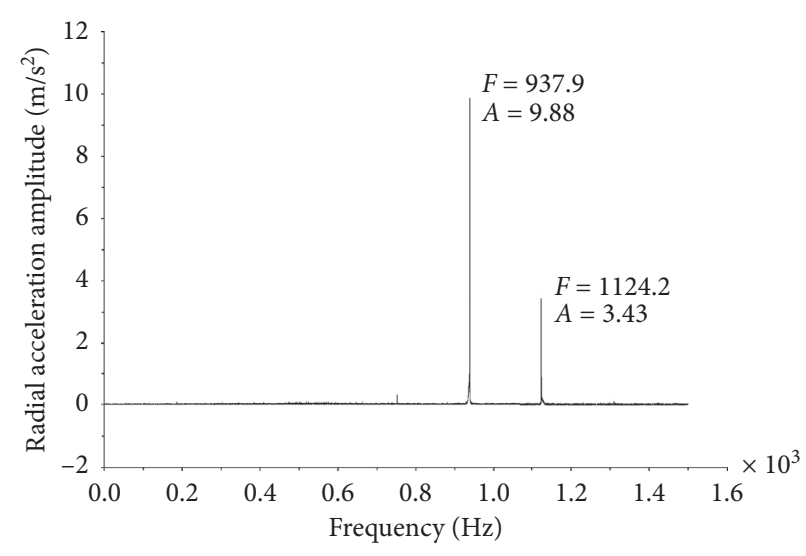

FIgURE 6: Radial acceleration of the original model.

TABle 3: Comparisons between simulations and the test.

\begin{tabular}{lcc}
\hline Direction & Simulation $\left(\mathrm{m} / \mathrm{s}^{2}\right)$ & Experiment $\left(\mathrm{m} / \mathrm{s}^{2}\right)$ \\
\hline Axial & 9.17 & 8.18 \\
Vertical & 20.49 & 21.65 \\
Radial & 9.88 & 15.29 \\
& Significant excitation frequency: & $937.9 \mathrm{~Hz}$ \\
\hline
\end{tabular}

TAвLE 4: Gear mesh frequencies of original parallel-axis helix stage.

\begin{tabular}{lccc}
\hline Condition & $\begin{array}{c}\text { Input } \\
\text { rotation (rpm) }\end{array}$ & $\begin{array}{c}\text { Output } \\
\text { rotation (rpm) }\end{array}$ & $\begin{array}{c}\text { Meshing } \\
\text { frequency }(\mathrm{Hz})\end{array}$ \\
\hline In & 8.989 & 1015 & 524.42 \\
Rated & 16 & 1806.72 & 933.47 \\
Out & 18.243 & 2060 & 1064.33 \\
& The actual ratio of the original model is 112.92 \\
\hline
\end{tabular}

validate that the excitation frequencies on rated work conditions are significantly different from any eigenfrequency; otherwise, a complicated forced oscillation including potential resonance would arise at a particular rotation velocity. Table 5 lists the segmental eigenfrequencies of the original model.

The modal analysis result of the original model highlights that the eigenfrequency of mode 98 is $937.3 \mathrm{~Hz}$, which is very close to the meshing frequency of the parallel-axis helix stage $(937.9 \mathrm{~Hz})$. The phenomenon indicates the existence of potential resonance in the gearbox drive train. The resonance deriving from the helix gear stage can sharply amplify the vibration responses of the HSS and must be the dominant mechanism of the terrible vibration of the faulted wind turbine gearbox. A reliable solution for reducing the resonance effect is to regulate the excitation frequency, because it is difficult to control the eigenfrequencies of the drive train; the regulation can be achieved by modifying the original design parameters of the helix gear stage (especially the numbers of teeth) to vary the meshing frequency. To validate the proposed strategy, a "modified model" with modified geometric parameters of the helix gear stage is proposed to study regulated HSS vibrations. 
TABle 5: Original eigenfrequencies (segmental).

\begin{tabular}{lcc}
\hline Mode & Damping coefficient & Eigenfrequency $(\mathrm{Hz})$ \\
\hline 96 & 0.0508 & 872.5 \\
97 & 0.0354 & 893.8 \\
98 & 0.0292 & 937.3 \\
99 & 0.0230 & 1014.5 \\
100 & 0.0303 & 1078.1 \\
\hline
\end{tabular}

3.2. Modified Model. Equation (11) indicates that the meshing frequency of parallel-axis helix stage can be changed by adjusting the numbers of teeth (the rotation velocities should be constant), and other geometric parameters of the gears should also be modified accordingly. The modified geometric parameters of the helix gear stage should be defined according to the following regulations:

(i) The meshing frequency of the stage should be regulated to maintain a significant distance from any eigenfrequency

(ii) The gear ratio is basically consistent with the original model

(iiii) The centre distance is absolutely the same as the original model

(iiv) The module of the gear pair is permitted to be regulated

(v) The strength of the teeth must satisfy essential requirements

The primary purpose of the modification is to regulate the numbers of teeth to alter the gear meshing frequencies, so other parameters, such as the shift factors, can also be varied slightly to maintain the gear geometry. After the modification, the modified gear parameters are listed in Table 6 and compared with the original model, and the modified meshing frequencies are listed in Table 7.

Table 8 lists the segmental eigenfrequencies of the modified model.

Tables 7 and 8 highlight that the theoretical modified meshing frequency of the helix gear stage on rated work conditions is changed to $720.3 \mathrm{~Hz}$, and the closest eigenfrequencies to the modified meshing frequency $(720.3 \mathrm{~Hz})$ are $670.7 \mathrm{~Hz}$ and $777.4 \mathrm{~Hz}$. The frequency distribution presents sufficient differences between the excitation frequency and the eigenfrequencies. The potential resonance effect should be weakened due to the modified geometric parameters of the gears, and the HSS vibration should be decreased effectively. The discussion can be validated by calculating the peak translational accelerations on the basis of the modified model.

Figures 7-9 indicate that the most significant excitation frequency of the modified HSS translational acceleration responses varies to $728.4 \mathrm{~Hz}$; however, all of the peak translational accelerations are still greater than vibration limitation as well, and the effectiveness of the vibration reduction is not satisfactory. Table 9 compares the peak translational accelerations of the modified model with the original values, and the comparison indicates that the translational vibration responses of the HSS are decreased to
TABLE 6: Parameter comparisons between modified and original helical gears.

\begin{tabular}{lc}
\hline Original model & Modified model \\
\hline Numbers of teeth: $127 / 31$ & Numbers of teeth: $98 / 24$ \\
Shift factors: $-0.2358 / 0.2476$ & Shift factors: $-0.055 / 0.3409$ \\
Module: $7 \mathrm{~mm}$ & Module: $9 \mathrm{~mm}$ \\
Helix angle: $9^{\circ}$ & Helix angle: $10^{\circ}$ \\
\hline
\end{tabular}

some degree. The principle is that the meshing frequency of the helix gear stage does not approach certain eigenfrequencies by adjusting the numbers of teeth and the module. The modified simulation results validate that the potential resonance must be a significant source of the faulted vibration, and the influence can be reduced by modifying the geometric parameters of the gears. Actually, the eigenfrequencies of the multibody system are mainly relevant to the structure of drive train. The variation in the geometric parameters of the gears does not affect the eigenfrequencies significantly as long as the mass and rotary inertia of the gears are the same. Consequently, the gear parameters shall be designed considering modal analysis to avoid resonance.

The simulation results of the modified model also present some phenomena that must be discussed. The original results indicate that the significant excitation frequency is only the fundamental meshing frequency of the helix gear stage $(937.9 \mathrm{~Hz})$. The modified results indicate that the responses of the axial and radial accelerations have an additional significant excitation frequency, which is the double meshing frequency of the excitation helix gear stage $(1456.9 \mathrm{~Hz})$, and the fundamental meshing frequency $(728.4 \mathrm{~Hz})$ is also a significant frequency as in the original results. This phenomenon means that the gearbox is excited by the double meshing frequency of the helix gear stage more seriously after the modification of the geometric parameters of the gears, and the integrated vibration energy may be increased by an additional excitation frequency. The most important validation is that all of the HSS translational accelerations have not been controlled significantly to be less than the vibration limitation. According to the above discussions, it is clear that the faulted gearbox vibration cannot be decreased effectively by only adjusting the numbers of teeth and module of helix gear stage. Other geometric gear parameters should be considered to investigate the most effective solution for the vibration fault.

3.3. Optimal Model. An excessively large vibration acceleration always indicates a serious exciting load, and the load must derive from the dynamic meshing force of the gear pair, so the excessively large vibration acceleration could be controlled by limiting the gear meshing force. In this research, the ratio and centre distance of the HSS gear pair are not allowed to vary, and the most effective method to control the gear meshing force is to regulate the helix angle of the gear pair. Consequently, an "optimal model" with a larger helix angle than that in the modified model is proposed, and 
TAвLE 7: Gear mesh frequencies of modified parallel-axis helix stage.

\begin{tabular}{lccc}
\hline Condition & Input rotation $(\mathrm{rpm})$ & Output rotation $(\mathrm{rpm})$ & Meshing frequency $(\mathrm{Hz})$ \\
\hline In & 9.018 & 1015 & 406 \\
Rated & 16 & 1800.8 & 720.3 \\
Out & 18.303 & 2060 & 824 \\
& \multicolumn{2}{c}{ The actual ratio of the modified model is 112.55} & \\
\hline
\end{tabular}

TABLE 8: Modified eigenfrequencies (segmental).

\begin{tabular}{lcc}
\hline Mode & Damping coefficient & Eigenfrequency $(\mathrm{Hz})$ \\
\hline 86 & 0.0393 & 651.5 \\
87 & 0.0203 & 670.7 \\
88 & 0.0240 & 777.4 \\
89 & 0.1206 & 783.7 \\
90 & 0.0200 & 811.5 \\
\hline
\end{tabular}

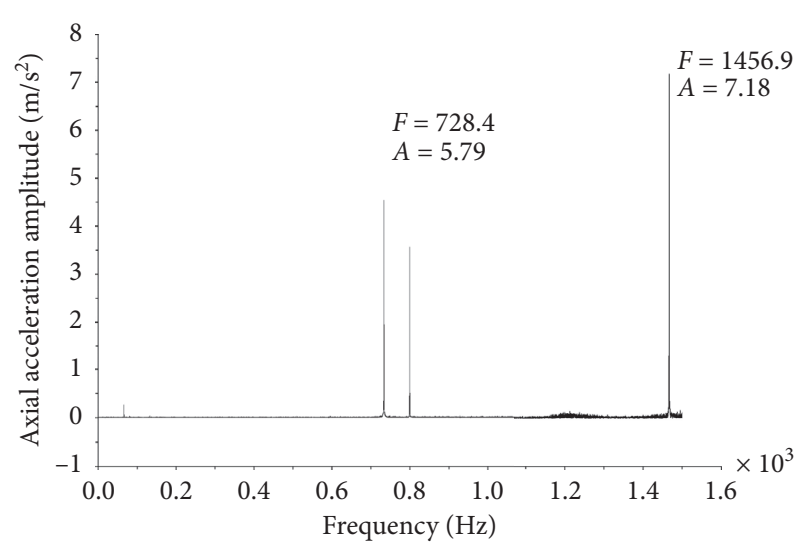

Figure 7: Axial acceleration of the modified model.

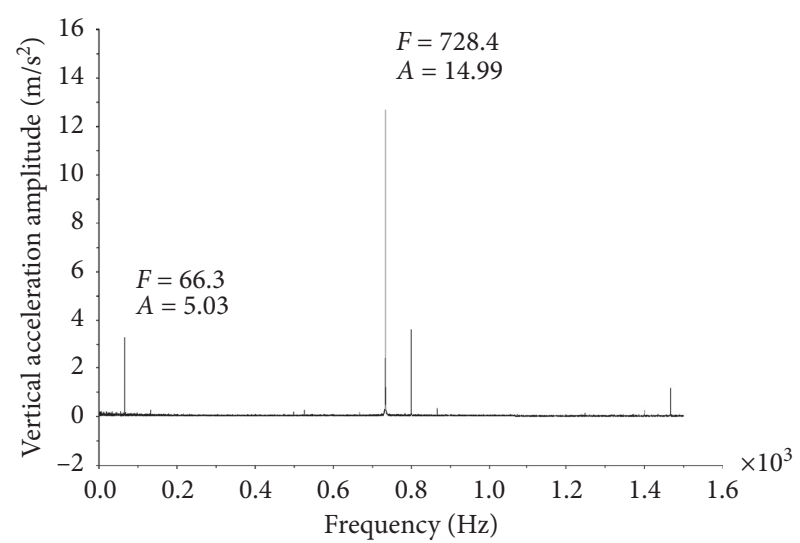

Figure 8: Vertical acceleration of the modified model.

the optimal geometric parameters of the helix stage should be defined according to the following regulations:

(i) The module of the gear pair is $9 \mathrm{~mm}$

(ii) The numbers of teeth are similar to the modified model

(iii) The gear ratio is basically consistent with the modified model

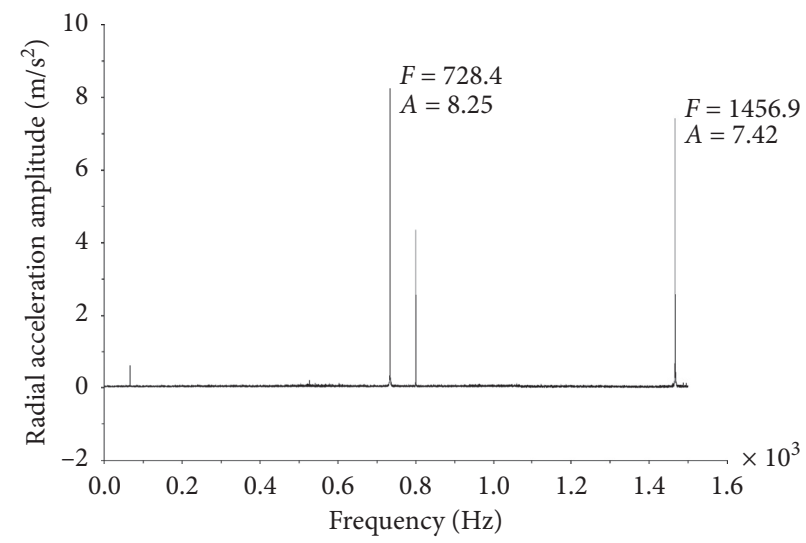

Figure 9: Radial acceleration of the modified model.

TABLE 9: Simulation result comparisons.

\begin{tabular}{lcc}
\hline Direction & Modified $\left(\mathrm{m} / \mathrm{s}^{2}\right)$ & Original $\left(\mathrm{m} / \mathrm{s}^{2}\right)$ \\
\hline Axial & 7.18 & 9.17 \\
Vertical & 14.99 & 20.49 \\
Radial & 8.25 & 9.88 \\
Excitation & $728.4 / 1456.9 \mathrm{~Hz}$ & $937.9 \mathrm{~Hz}$ \\
\hline
\end{tabular}

TABle 10: Parameter comparisons between optimal and modified helical gears.

\begin{tabular}{lc}
\hline Modified model & Optimal model \\
\hline Numbers of teeth: $98 / 24$ & Numbers of teeth: $90 / 22$ \\
Shift factors: $-0.055 / 0.3409$ & Shift factors: $0.1054 / 0.337$ \\
Module: $9 \mathrm{~mm}$ & Module: $9 \mathrm{~mm}$ \\
Helix angle: $10^{\circ}$ & Helix angle: $25^{\circ}$ \\
\hline
\end{tabular}

(iv) The centre distance is absolutely the same as the modified model

(v) The helix angle should be increased significantly

The primary purpose of the optimization is to regulate the helix angle significantly to investigate the relationships between the helix angle and HSS vibration; other parameters, such as the shift factors, can also be varied slightly to maintain the gear geometry. After the optimization, the optimized gear parameters are listed in Table 10 and compared with those of the modified model, and the optimized meshing frequencies are listed in Table 11.

Table 12 lists the segmental eigenfrequencies of the optimal model.

Tables 11 and 12 highlight that the theoretical optimal meshing frequency of the helix gear stage on rated work conditions is varied to $661.53 \mathrm{~Hz}$, and the eigenfrequencies 
TABLE 11: Gear mesh frequencies of optimal parallel-axis helix stage.

\begin{tabular}{lccc}
\hline Condition & Input rotation $(\mathrm{rpm})$ & Output rotation $(\mathrm{rpm})$ & Meshing frequency $(\mathrm{Hz})$ \\
\hline In & 9 & 1015 & 372.17 \\
Rated & 16 & 1804.16 & 661.53 \\
Out & 18.27 & 2060 & 755.33 \\
& & The actual ratio of the optimal model is 112.76 & \\
\hline
\end{tabular}

TABLE 12: Optimal eigenfrequencies (segmental).

\begin{tabular}{lcc}
\hline Mode & Damping coefficient & Eigenfrequency $(\mathrm{Hz})$ \\
\hline 85 & 0.0200 & 642.3 \\
86 & 0.0399 & 652.4 \\
87 & 0.0239 & 777.6 \\
88 & 0.1206 & 783.8 \\
89 & 0.0237 & 828.3 \\
\hline
\end{tabular}

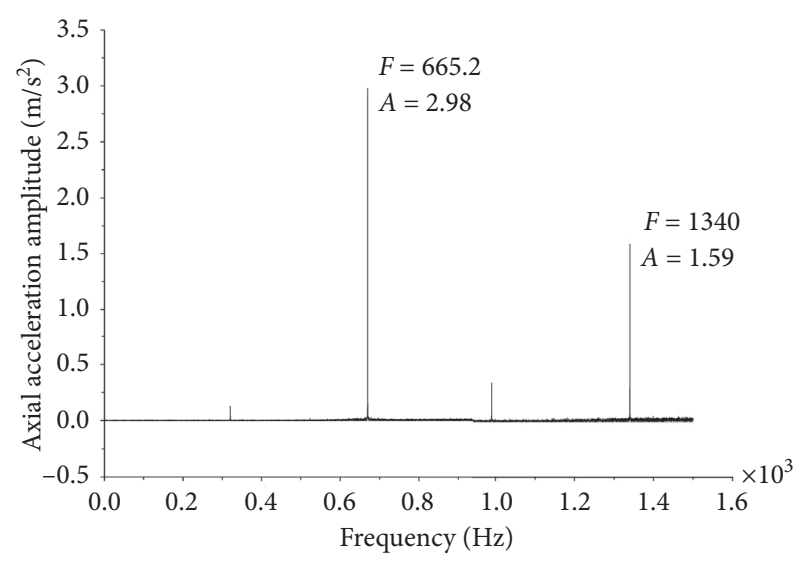

FIgURE 10: Axial acceleration of the optimal model.

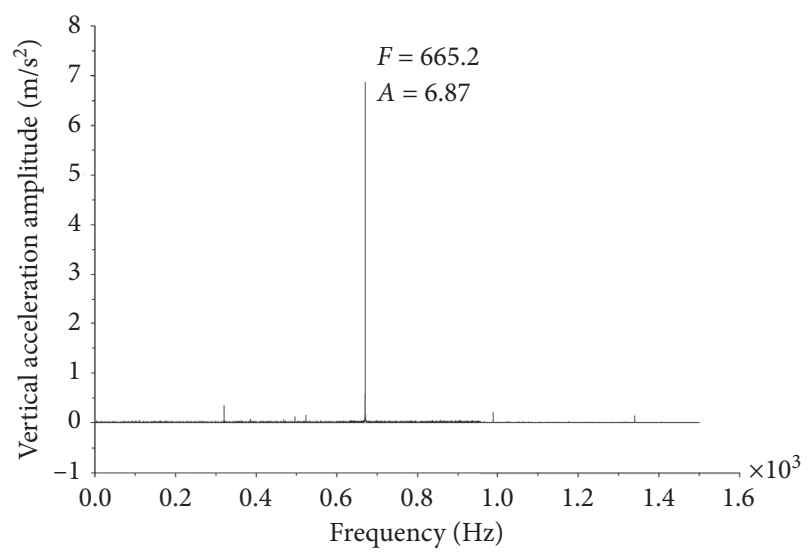

Figure 11: Vertical acceleration of the optimal model.

closest to the optimal meshing frequency $(661.53 \mathrm{~Hz})$ are $652.4 \mathrm{~Hz}$ and $777.6 \mathrm{~Hz}$. The differences between the excitation frequency and the eigenfrequencies are also sufficient as with the modified model, and the data listed in Tables 8 and 12 demonstrate that the geometric parameters of the gears do not affect the eigenfrequencies of the drive train significantly. To investigate the most effective solution for the vibration fault, the simulation results of the optimal

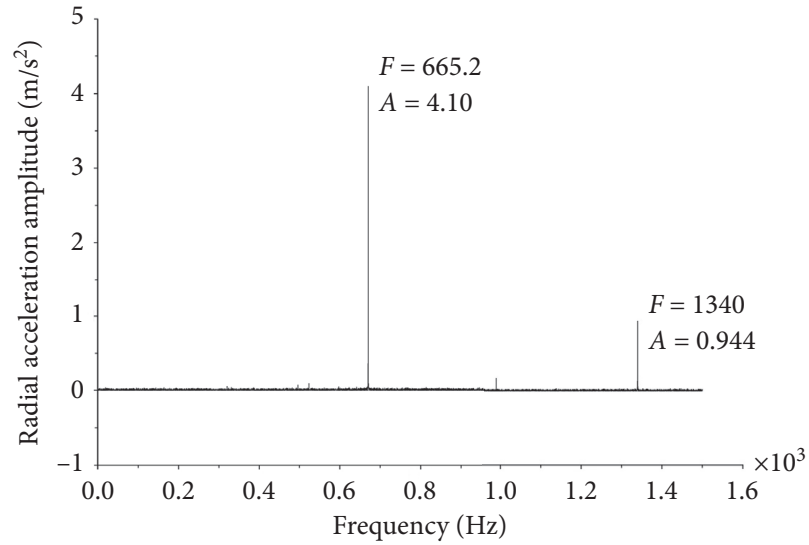

Figure 12: Radial acceleration of the optimal model.

TABLE 13: Simulation results comparisons (all models).

\begin{tabular}{lccc}
\hline Direction & Optimal $\left(\mathrm{m} / \mathrm{s}^{2}\right)$ & Modified $\left(\mathrm{m} / \mathrm{s}^{2}\right)$ & Original $\left(\mathrm{m} / \mathrm{s}^{2}\right)$ \\
\hline Axial & 2.98 & 7.18 & 9.17 \\
Vertical & 6.87 & 14.99 & 20.49 \\
Radial & 4.10 & 8.25 & 9.88 \\
Excitation & $665.2 \mathrm{~Hz}$ & $728.4 / 1456.9 \mathrm{~Hz}$ & $937.9 \mathrm{~Hz}$ \\
\hline
\end{tabular}

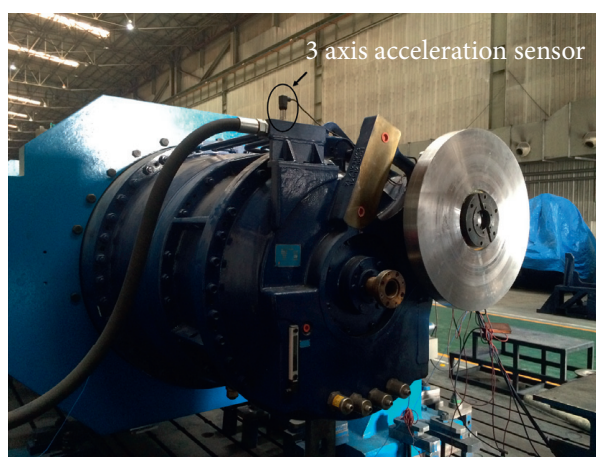

Figure 13: Primary test gearbox and sensor position.

model should be illustrated and discussed for a reliable validation.

Figures 10-12 indicate that the significant excitation frequency of the optimized HSS translational acceleration responses is changed to $665.2 \mathrm{~Hz}$, and all of the peak translational accelerations are less than vibration limitation. In this case study, the optimized simulation results show that the significant excitation frequency and the corresponding peak translational accelerations are obviously regulated, and the phenomenon signifies the vibration fault of the gearbox is solved. To summarize the effectiveness of the optimization 


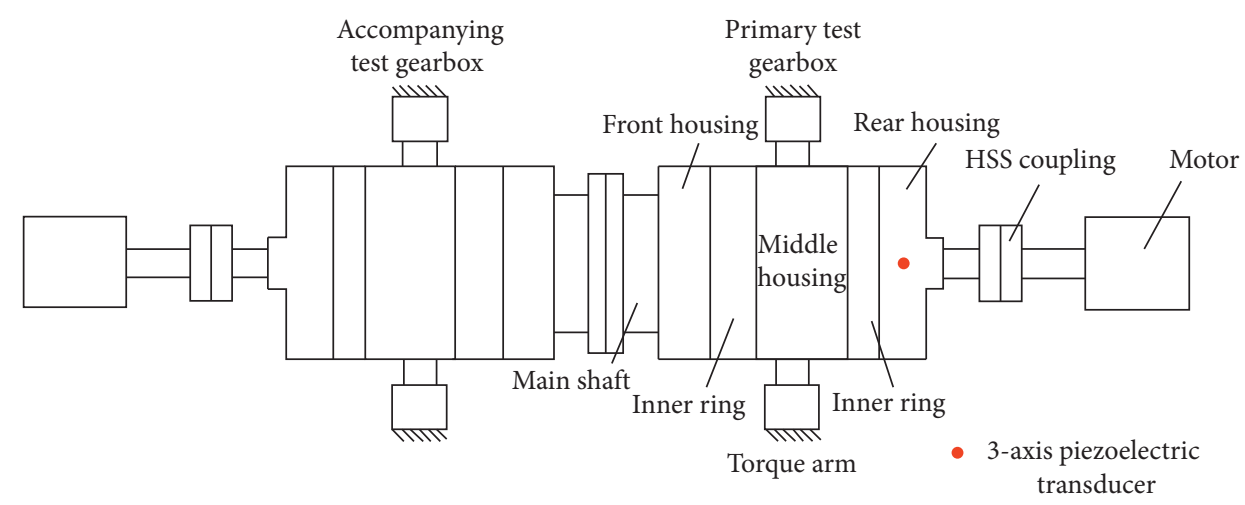

FIgURE 14: Back-to-back vibration test bench assembly [22].

according to the data in Table 13, the significant excitation frequency is decreased from $937.9 \mathrm{~Hz}$ (original model) to $728.4 \mathrm{~Hz}$ (modified model) owing to the variation of the numbers of teeth and significantly altered to $665.2 \mathrm{~Hz}$ (optimal model) by increasing the helix angle of the gear pair. The peak vertical acceleration of the optimal model is finally decreased by $66.47 \%$ compared with that of the original model; the peak axial and radial accelerations of the optimal model are finally decreased by $67.5 \%$ and $58.5 \%$, respectively, compared with those of the original model. After the optimal solutions, the comparisons indicate that the vibration behaviour of the HSS has been optimized to an expected level, implying that the current vibration load (dynamic gear meshing force) is acceptable, and the life and the reliability of the parallel-axis helix stage can be improved significantly. The mechanism of the vibration reduction in this case study is a reduction in the resonance effects on the HSS vibration by regulating the excitation frequency. The most highlighted distinctions among the original model, modified model, and optimal model are the alteration of the number of teeth and helix angle (see Tables 6 and 10), so it is clear that the most significant factor for solving the vibration fault is the geometric parameters of the helix gear stage.

The comparisons of different simulation results have demonstrated that the HSS vibrations are definitely influenced by the geometric parameters of the gears, which can be controlled effectively by adjusting the meshing frequency and increasing helix angle of the helix gear stage. For the purpose of vibration reduction, the numbers of teeth of gears must be decided critically to primarily reduce potential resonance risk; the modal analysis results indicated that the alteration of geometric gear parameters could not affect the eigenfrequencies of the drive train significantly, so the helix angle of the gear pair should be designed to be as large as possible to limit an excessively large gear meshing force. The process of the proposed optimizations implies that the detailed parameters of any factor that is relevant to the vibration behaviour of the gearbox should be designed considering the resonance effect and dynamic meshing load. This deduction is an important principle for solving the vibration fault of the wind turbine gearbox, and the effectiveness of the solution can be validated by the vibration test based on the optimized prototype.

\section{Experimental Validation}

A type of the back-to-back vibration test bench for wind turbine gearbox was assembled for experimental validation, and the optimized helix gears were manufactured to replace the original faulted gears. To consider the interchangeability of the parallel-axis helix stage, the central distance of the optimized helix gear stage should be identical with the original design scheme by altering some other geometric gear parameters and it is very significant to guarantee the primary performance of the gearbox and reduce the wasted maintenance costs. The vibration test for the optimized gearbox is carried out to monitor the translational accelerations of HSS, which can be reflected on the rear housing of the gearbox. Figure 13 illustrates that a 3 -axis piezoelectric transducer was placed on the rear housing to record translational accelerations. To guarantee the experimental results are adequately precise for further evaluation, the testing process was repeated five times for calculating average directional accelerations. Figure 14 illustrates the vibration test bench assembly as follows.

The original vibration test result based on faulted gearbox also derives from the identical test bench and program. The experimental results validate that the serious translational vibrations on the rear housing are caused by potential resonance in the parallel-axis helix stage, and the resonance effects can be reduced by enlarging the helix angle of the gear pair and regulating the meshing frequency. Finally, the entire gearbox vibrations are decreased effectively by replacing the parallel-axis helix gear pair.

Table 14 displays the experimental translational acceleration results, and the values are means of five vibration tests involving the gearbox with the optimal helix gear stage (the optimized gearbox). The data are considered to be the most reliable experimental results for expected validation. Table 15 compares current vibrations of the optimized gearbox with original vibrations of the faulted gearbox, and Table 16 shows the comparisons between the optimal simulation results and the experimental data of the optimized gearbox. All of the data display that the faulted gearbox vibrations have been decreased to less than required limitation. This result validates the effectiveness of the proposed solution and the significance of the gear parameter 
TABLE 14: Vibration test results after optimization.

\begin{tabular}{lc}
\hline Direction & Acceleration $\left(\mathrm{m} / \mathrm{s}^{2}\right)$ \\
\hline Axial & 2.257 \\
Vertical & 6.184 \\
Radial & 4.638 \\
\hline
\end{tabular}

TABLE 15: Comparison of the experimental results.

\begin{tabular}{lcc}
\hline Direction & Optimized $\left(\mathrm{m} / \mathrm{s}^{2}\right)$ & Original $\left(\mathrm{m} / \mathrm{s}^{2}\right)$ \\
\hline Axial & 2.257 & 8.18 \\
Vertical & 6.184 & 21.65 \\
Radial & 4.638 & 15.29 \\
\hline
\end{tabular}

TABLE 16: Comparisons between simulations and test (the optimal model).

\begin{tabular}{lcc}
\hline Direction & Simulation $\left(\mathrm{m} / \mathrm{s}^{2}\right)$ & Experiment $\left(\mathrm{m} / \mathrm{s}^{2}\right)$ \\
\hline Axial & 2.98 & 2.257 \\
Vertical & 6.87 & 6.184 \\
Radial & 4.10 & 4.638 \\
\hline
\end{tabular}

optimization and also demonstrates that some additional vibration behaviour due to the resonance effect can be controlled by increasing the helix angle of the gears and adjusting the numbers of teeth. These parameters are the most significant factors that are relevant to excessively large translational vibrations of the original faulted gearbox.

\section{Conclusions}

This article describes a case study that investigates the effects of some basic parameters of a parallel-axis helix gear stage on wind turbine gearbox vibration. A coupled multibody dynamic model for a faulted wind turbine gearbox drive train with serious vibrations is constructed and used in the research. According to the results of the dynamic responses derived from the simulation, the relevant discussions lead to the following conclusions:

(i) The potential resonance effect and excessively large exciting load in parallel-axis helix stage cause the serious vibration of the faulted wind turbine gearbox

(ii) The numbers of teeth of the gear pair are relevant to the existence of potential resonance, and the helix angle will directly influence the exciting load

(iii) The numbers of teeth should be decided with consideration of the modal analysis results, which should ensure that the excitation frequencies for important input rotation velocities do not correspond to any eigenfrequencies to avoid potential resonance

(iv) The effective solution for the faulted wind turbine gearbox should include two parts: (1) an optimization of the numbers of teeth of the parallel-axis helix gear pair to avoid resonance risk and (2) an increased helix angle to optimize the dynamic gear meshing force to limit excitation
For the case study in this article, the accuracy and reliability of the proposed solutions have been validated by multiple prototype vibration tests, and the conclusions can be considered as a common principle for solving similar vibration problems of other faulted gearboxes. In addition, the conclusions and methodology of this research are also significant for controlling potential vibration faults of gearbox prototypes. If designers always consider the eigenfrequencies of the drive train and dynamic gear meshing force optimization when defining the important gear parameters, the dynamic vibration of the gearbox prototype can be predicted and optimized actively during the theoretical design process. The methodology is very beneficial for improving the reliability of the gearbox prototype and reducing the manufacturing costs.

In future work, a "virtual prototype vibration test bench" will be developed on the basis of the multibody dynamic simulation, which can simulate the practical boundary conditions of an actual test bench. The finished gearbox model can be assembled using the virtual test bench to evaluate its dynamic performance, and the significant parameters can also be regulated conveniently to find optimal values before manufacturing. Consequently, the vibration of an actual prototype can be controlled effectively by the methodology, which is beneficial for avoiding vibration problems such as the faulted wind turbine gearbox considered in this case study.

\section{Nomenclature}

\section{Subscript}

$c, p, s, r$ : Carrier $(c)$, planet $(p)$, sun $(s)$, and ring $(r)$

$p j$ : $\quad$ The planet gear number in a planetary stage $(j=1$, 2,3)

$i$ : $\quad$ The gear stage number $(i=1,2,3)$

Items

$m_{a}, m_{a b}: \quad$ The single mass in the drive train $(a=1,2$,

$F_{K}, F_{I}, F_{C}$

$q(t):$

$q_{b}(t):$

$Q_{a}(t):$

$K_{a}, K_{a b}$

$C_{a}, C_{a b}$ $\ldots, n, b=1,2, \ldots, n$ )

$[m],[C],[K]$ :

Elastic force, inertial force, and damping force

Displacement function of $m_{a}$

Displacement function of each $m_{a}$ in the drive train $(b=1,2, \ldots, n)$

The exciting force acting on mass $m_{a}$ $(a=1,2, \ldots, n)$

Stiffness of mass $m_{a}$ and $m_{a b}$ and damping of mass $m_{a}$ and $m_{a b}$

$\{q(t)\} /[u]: \quad$ The generalized displacement vectors

$\{Q(t)\} /(F): \quad$ The generalized force vectors

$\alpha_{1 s}, \alpha_{1 p}, \alpha_{2 s}, \alpha_{2 p}$ : Working pressure angles of the sunplanet meshing and planet-ring meshing in stage 1 and stage 2

$X_{1 s p}, X_{2 s p}$ : The integrated meshing force between the sun and planet in stage 1 and stage 2

$X_{1 r p}, X_{2 r p}$ : The integrated meshing force between the planet and ring in stage 1 and stage 2

$F_{i s p j:}$
The meshing forces between sun gear and planet gear $j$ in stage $i(i=1,2, j=1,2,3)$ 


$\begin{array}{ll}F_{i r p j}: & \text { The meshing forces between ring and } \\ & \text { planet gear } j \text { in stage } i \\ F_{3 w h s}: & \text { The meshing forces between wheel gear } \\ & \text { and pinion gear in stage } 3 \\ r_{i c}: & \text { Rotating radius of the carrier in } i \text { stage } \\ & \left(i=1,2,3, k=s, r, p_{1}, p_{2}, p_{3}, w, h s\right) \\ r_{i k}: & \text { Base radius of the gears in } i \text { stage } \\ K_{i c}, C_{i c}: & \text { Stiffness/damping of the carriers in stage } i \\ & (i=1,2) \\ K_{1 s 2 c}, C_{1 s 2 c}, & \text { Stiffness and damping of the spline } \\ K_{2 s 3 w}, C_{2 s 3 w}: & \text { between the stage } 1 \text { sun and stage } 2 \\ & \text { carrier, and stage } 2 \text { sun and stage } 3 \text { wheel } \\ T_{\mathrm{in}}, T_{\text {out }}: & \text { gear } \\ f_{i}: & \text { Input and output torque } \\ m_{i k}: & \text { The ratio of stage } i(i=1,2,3) \\ & \text { Mass of the component }(i=1,2,3, k=c, s, \\ u_{i k}: & \left.p_{1}, p_{2}, p_{3}, w, h s\right) \\ \omega_{i}: & \text { Displacement of the component } \\ \varphi_{i}: & \text { Undamped eigenfrequency of mode } i \\ \omega_{d i}: & \text { The mode shape of mode } i \\ \xi_{i}: & \text { Damped eigenfrequency of mode } i \\ f_{\text {mesh }}: & \text { Damping coefficient } \\ n_{a}, n_{b}: & \text { Tear meshing frequency } \\ Z_{a}, Z_{b}: & \text { The rotation velocities of the gears } \\ & \text { The numbers of teeth of the gears. }\end{array}$

\section{Data Availability}

Some or all data, models, or code generated or used during the study are available in a repository or online in accordance with funder data retention policies.

\section{Conflicts of Interest}

The authors declare that there are no conflicts of interest regarding the publication of this article.

\section{Authors' Contributions}

All authors contributed to the study conception and design. Material preparation, data collection, and analysis were performed by all authors. The first draft of the manuscript was written by Zheng $\mathrm{Li}$, and all authors commented on previous versions of the manuscript. All authors read and approved the final manuscript.

\section{Acknowledgments}

The research in this article was supported by the Dalian Huarui Heavy Industry Group Co., Ltd. and funded by Dalian Maritime University.

\section{References}

[1] W. Hu, Z. Liu, D. Liu, and X. Hai, "Fatigue failure analysis of high speed train gearbox housings," Engineering Failure Analysis, vol. 73, pp. 57-71, 2017.

[2] A. Romero, Y. Lage, S. Soua, B. Wang, and T.-H. Gan, "Vestas V90-3MW wind turbine gearbox health assessment using a vibration-based condition monitoring system," Shock and Vibration, vol. 2016, pp. 1-18, 2016.

[3] M. Tosun, M. Yıldız, and A. Özkan, "Investigation and refinement of gearbox whine noise," Applied Acoustics, vol. 130, pp. 305-311, 2018.

[4] W. Lu, Y. Zhang, H. Cheng, Y. Zhou, and H. Lv, "Research on dynamic behavior of multistage gears-bearings and box coupling system," Measurement, vol. 150, p. 107096, 2020.

[5] J. Parra and C. M. Vicuña, "Two methods for modeling vibrations of planetary gearboxes including faults: comparison and validation," Mechanical Systems and Signal Processing, vol. 92, pp. 213-225, 2017.

[6] G. He, K. Ding, X. Wu, and X. Yang, "Dynamics modeling and vibration modulation signal analysis of wind turbine planetary gearbox with a floating sun gear," Renewable Energy, vol. 139, pp. 718-729, 2019.

[7] M. Zhao and J. C. Ji, "Nonlinear torsional vibrations of a wind turbine gearbox," Applied Mathematical Modelling, vol. 39, no. 16, pp. 4928-4950, 2015.

[8] H. Al-Hamadani, T. An, M. King, and H. Long, "System dynamic modelling of three different wind turbine gearbox designs under transient loading conditions," International Journal of Precision Engineering and Manufacturing, vol. 18, no. 11, pp. 1659-1668, 2017.

[9] Z. Li, B. Wen, K. Wei, W. Yang, Z. Peng, and W. Zhang, "Flexible dynamic modeling and analysis of drive train for Offshore Floating Wind Turbine," Renewable Energy, vol. 145, pp. 1292-1305, 2020.

[10] J. Tan, C. Zhu, C. Song, Y. Li, and X. Xu, "Dynamic modeling and analysis of wind turbine drivetrain considering platform motion," Mechanism and Machine Theory, vol. 140, pp. 781-808, 2019.

[11] X. Chen, Y. Yang, Z. Cui, and J. Shen, "Vibration fault diagnosis of wind turbines based on variational mode decomposition and energy entropy," Energy, vol. 174, pp. 1100-1109, 2019.

[12] N. Barbieri, G. de Sant'Anna Vitor Barbieri, B. M. Martins, L. de Sant'Anna Vitor Barbieri, and K. F. de Lima, "Analysis of automotive gearbox faults using vibration signal," Mechanical Systems and Signal Processing, vol. 129, pp. 148-163, 2019.

[13] Y. Kong, T. Wang, and F. Chu, "Meshing frequency modulation assisted empirical wavelet transform for fault diagnosis of wind turbine planetary ring gear," Renewable Energy, vol. 132, pp. 1373-1388, 2019.

[14] W. Teng, X. Ding, H. Cheng, C. Han, Y. Liu, and H. Mu, "Compound faults diagnosis and analysis for a wind turbine gearbox via a novel vibration model and empirical wavelet transform," Renewable Energy, vol. 136, pp. 393-402, 2019.

[15] S. Wei, J. Zhao, Q. Han, and F. Chu, "Dynamic response analysis on torsional vibrations of wind turbine geared transmission system with uncertainty," Renewable Energy, vol. 78, pp. 60-67, 2015.

[16] S. Wang, C. Zhu, C. Song, H. Liu, J. Tan, and H. Bai, "Effects of gear modifications on the dynamic characteristics of wind turbine gearbox considering elastic support of the gearbox," Journal of Mechanical Science and Technology, vol. 31, no. 3, pp. 1079-1088, 2017.

[17] P. Yi, P. Huang, and T. Shi, "Numerical analysis and experimental investigation of modal properties for the gearbox in wind turbine," Frontiers of Mechanical Engineering, vol. 11, no. 4, pp. 388-402, 2016.

[18] A. Tatar, C. W. Schwingshackl, and M. I. Friswell, "Dynamic behaviour of three-dimensional planetary geared rotor systems," Mechanism and Machine Theory, vol. 134, pp. 39-56, 2019. 
[19] Y. Lei, L. Hou, Y. Fu, J. Hu, and W. Chen, "Research on vibration and noise reduction of electric bus gearbox based on multi-objective optimization," Applied Acoustics, vol. 158, no. 15 , p. $107037,2020$.

[20] V. Hiremath and N. Venkataram, "Vibration charecteristics of a high speed gearbox through dynamic analysis," Materials Today: Proceedings, vol. 4, no. 10, pp. 10935-10943, 2017.

[21] J. G. Wang, Y. Wang, and Z. P. Huo, "Establishment of mathematic model and study of vibration modes for multistage planetary gear train of wind turbine gearboxes," Sichuan University (Engineering Science Edition), vol. 45, no. 3, pp. 189-196, 2013.

[22] Z. Li and Y. Zhou, "Research on gearbox dynamic design methodology based on modal optimization," Journal of Vibroengineering, vol. 22, no. 1, pp. 156-169, 2020. 